\title{
Temozolomide in the Treatment of Aggressive Pituitary Tumors- An Overview of Existing Knowledge and Future Perspectives
}

\author{
Ann McCormack, MBBS, FRACP, PhD \\ Staff Specialist Endocrinology, St Vincent's Hospital and Garvan Institute of Medical Research, Sydney, Australia
}

\begin{abstract}
The management of aggressive pituitary tumors remains a challenge, however, the recent identification of temozolomide as a chemotherapeutic agent with significant efficacy against these tumors has heralded a new therapeutic era. There has been an exponential growth in the international experience with temozolomide over the past five years, now totaling 50 published cases. Overall, $67 \%$ of cases demonstrated a response to temozolomide. Prolactin- and adrenocorticotrophic hormone (ACTH)-secreting tumors respond more frequently than non-functioning tumors. Response is typically evident in the first three months of treatment. Adverse effects occur in almost half of patients, although the majority are mild. The expression of a DNA repair enzyme, 06-methylguanine-DNA methyltransferase (MGMT), as determined by immunohistochemistry, appears to be the primary determinant of response to temozolomide in pituitary tumors. There is suggestion that MGMT may also play a role in pituitary tumorigenesis. Over the next few years we will see temozolomide used earlier in the treatment algorithm of aggressive pituitary tumors, making it imperative to collect global long-term data on its use.
\end{abstract}

\section{Keywords}

Pituitary tumor, pituitary carcinoma, temozolomide, MGMT

Disclosure: The author has no conflicts of interest to declare.

Received: July 2, 2012 Accepted: August 21, 2012 Citation: US Endocrinology, 2012;8(2):112-7 DOI: 10.17925/USE.2012.08.02.112

Correspondence: Ann McCormack, MBBS, FRACP, PhD, Department of Endocrinology, Garvan Institute of Medical Research, 384 Victoria Street, Darlinghurst, NSW 2010,

Australia. E: a.mccormack@garvan.org.au

Pituitary tumors are common, with an estimated prevalence of $16.7 \%$ in the general population based on imaging and autopsy studies. ${ }^{1}$ Clinically significant pituitary tumors are more prevalent than previously recognized, with one case per 1,000-1,300 people., The majority of pituitary tumors are indolent, slow-growing neoplasms. However, 40-50 \% of pituitary tumors are locally invasive and commonly unable to be completely surgically excised. ${ }^{4,5}$ An 'aggressive pituitary tumor' typically refers to an invasive pituitary tumor that demonstrates progressive growth despite multimodal therapy, including surgery, and radiotherapy. Whilst these tumors have malignant potential, the term pituitary carcinoma is strictly reserved for those tumors with demonstrated craniospinal or systemic metastases. ${ }^{6}$ As there is a lack of formal criterion used to define an aggressive pituitary tumor, epidemiologic data with respect to this group is lacking. The World Health Organization pathological classification of an 'atypical pituitary adenoma' (Ki67 >3\%, excessive p53 immunoreactivity and increased mitotic activity) was coined in an attempt to identify a tumor with the potential for more aggressive behaviour. ${ }^{7}$ A recent study, conducted in a tertiary referral center, identified $15 \%$ of atypical adenomas amongst their surgical cohort. ${ }^{8}$ Pituitary carcinoma is rare, accounting for $0.2 \%$ of pituitary tumors. ${ }^{9}$

The management of aggressive pituitary tumors is challenging, and there is substantial morbidity and mortality associated with both the tumor and treatment. Patients often undergo multiple surgeries and radiotherapy in an attempt to control tumor growth. These tumors are often also resistant to medical therapies, such as dopamine agonists. Historically, systemic chemotherapy was reserved as a 'last resort' therapy, principally due to the lack of identification of a consistently effective chemotherapeutic agent. However, over the past five years temozolomide, an oral alkylating agent commonly used in the management of glioblastoma, has emerged as the first chemotherapeutic with substantial efficacy in the treatment of aggressive pituitary tumors. International experience with temozolomide, as used in the management of an aggressive pituitary tumor, has grown exponentially, with 50 published cases now reported. This review will summarize and examine the existing knowledge base, but also speculate on the future possibilities regarding the use of temozolomide in pituitary tumors. The role of 06-methylguanine-DNA methyltransferase (MGMT), a DNA repair protein, as a biomarker of response to temozolomide and its possible role in pituitary tumor biology will be discussed.

\section{Temozolomide and DNA Repair systems}

Temozolomide is an oral second-generation alkylating agent of the imidotetrazine class. ${ }^{10}$ The lipophilic nature of the temozolomide molecule promotes efficient passage across the blood-brain barrier, advantageous when treating pituitary tumors. Conversion of temozolomide to a highly reactive methyldiazonium ion is responsible for the formation of methyl adducts with DNA purinic bases. ${ }^{11}$ Figure 1 illustrates the mechanism 


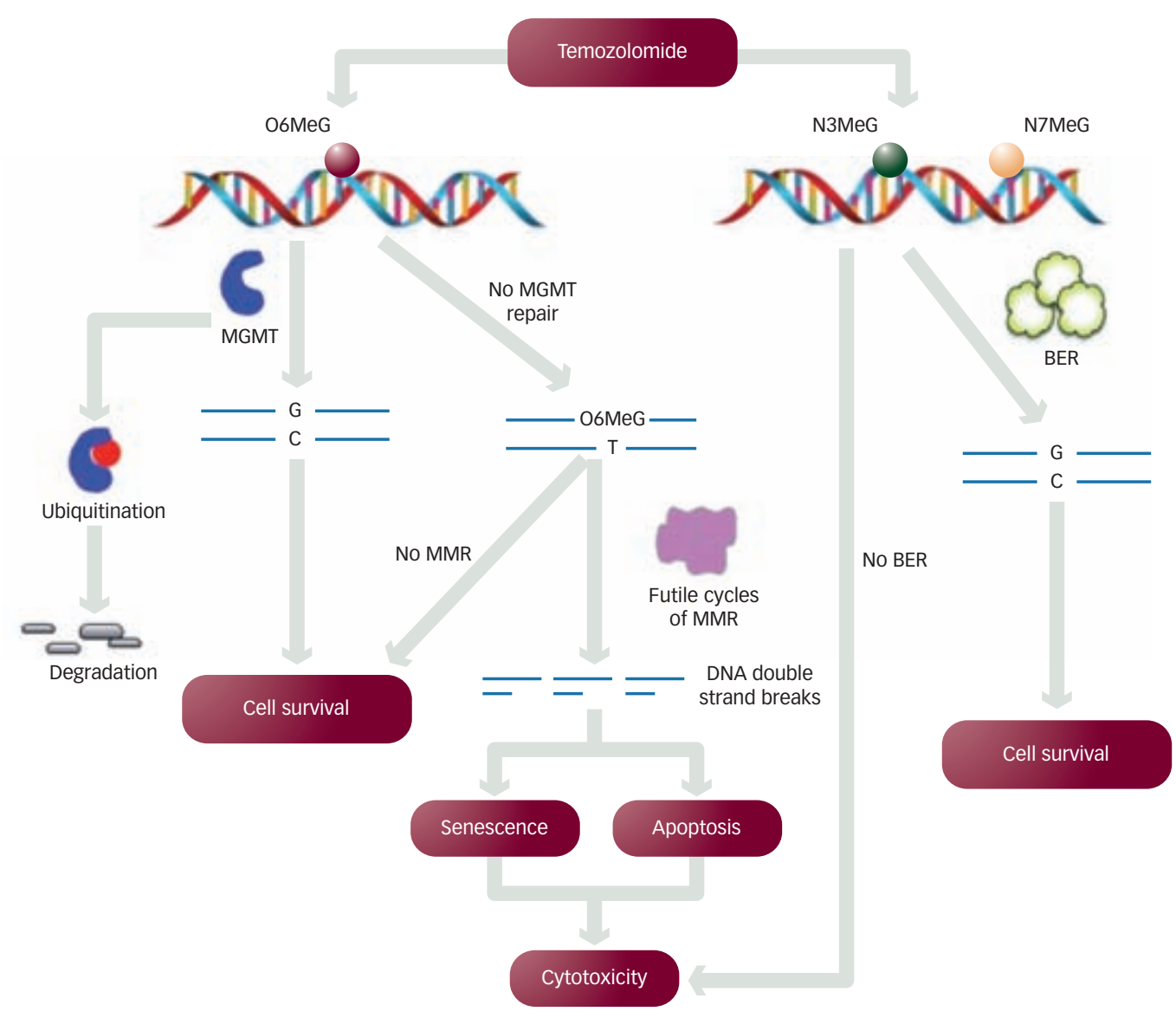

O6MeG = 06-methylated guanine; $B E R=$ base excision repair; $M G M T=06$-methylguanine-DNA methyltransferase; $M M R=$ mismatch repair; N3MeG = N3-methylguanine; N7MeG $=$ N7-methylguanine

of temozolomide cytotoxicity. The critical methyl adduct produced by temozolomide is the lesion at 06-guanine (06MeG). 06MeG adducts account for only $5 \%$ of alkylating lesions induced by temozolomide but deliver the greatest cytotoxicity. Other alkylating lesions induced by temozolomide are N3-methylguanine (N3MeG) and N7-methylguanine (N7MeG). In the presence of the base excision repair (BER) pathway, N3MeG and N7MeG lesions are repaired. MGMT is responsible for repairing $06 \mathrm{MeG}$ lesions. In the absence of MGMT, methylated guanine lesions incorrectly pair with thymine, triggering the mismatch repair (MMR) pathway. In the presence of MMR activation there is incorrect reinsertion of thymine opposite the $06 \mathrm{MeG}$ lesion resulting in futile cycles of repair and ultimately DNA strand breaks. This is followed by either activation of the apoptotic cascade or a senescence-like state. ${ }^{11,12}$

The standard temozolomide dosing regime is $200 \mathrm{mg} / \mathrm{m}^{2}$ given daily for five days every 28 days. ${ }^{13}$ Common alternative regimes include 'dose-dense' protocols, such as $150 \mathrm{mg} / \mathrm{m}^{2}$ days 1-7 and days 14-21 of a 28 day cycle, and 'metronomic' protocols using continuous daily low-dose (50-75 mg/m²) temozolomide. These alternative dosing schedules have been reported to be effective in patients with progressive or recurrent gliomas, although this has been recently disputed. ${ }^{10,14}$ Temozolomide is standard of care in the management of glioblastoma and advanced melanoma, and has demonstrated efficacy for malignant neuroendocrine tumors. ${ }^{15,16}$ In glioblastoma, temozolomide monotherapy is typically administered for six months following six weeks of concurrent radiotherapy and low-dose temozolomide..$^{15}$ However, improved survival rates have been reported with use of temozolomide for 12-24 months, and there are cases of sustained remissions with continuous treatment out to eight years. ${ }^{17,18}$ Temozolomide is generally well tolerated as compared with most chemotherapeutic agents. Adverse effects are commonly mild and include nausea, vomiting, and fatigue. Myelosuppression is also common and dose-limiting, particularly lymphopenia and thrombocytopenia, however, severe myelosuppression leading to discontinuation of temozolomide is rare. Dose-dense regimes have been associated with an increased myelotoxicity and risk of opportunistic infection. ${ }^{10}$ Rare reports of myelodysplastic syndrome, aplastic anemia, and lymphoma have been described following temozolomide therapy. ${ }^{19-21}$ Other rare adverse events reported include hypersensitivity pneumonitis and hearing loss. ${ }^{22,23}$

\section{Collective Experience with Temozolomide in Pituitary Tumors}

The first reports describing the successful use of temozolomide in pituitary tumors were published in 2006. ${ }^{24,25}$ These early cases were pituitary carcinomas, but it was not long before significant responses were also reported for locally aggressive pituitary adenomas. ${ }^{26-30}$ There are now 50 published reports, both carcinomas and aggressive adenomas, in 


\section{Figure 2: Clinical Spectrum of Temozolomide Response}
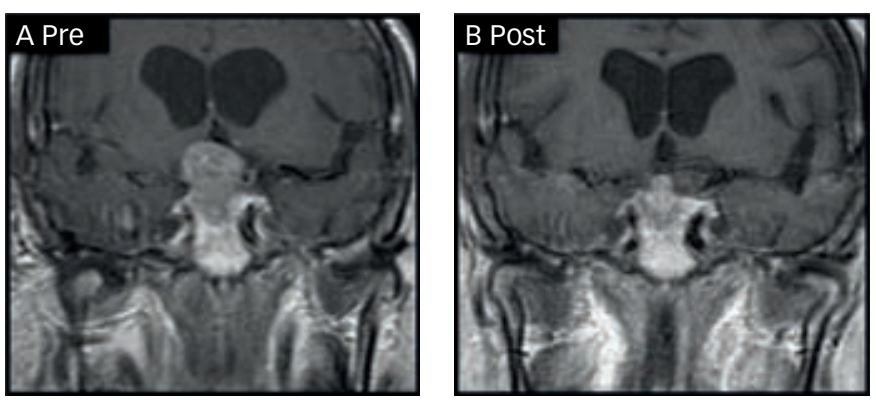

C

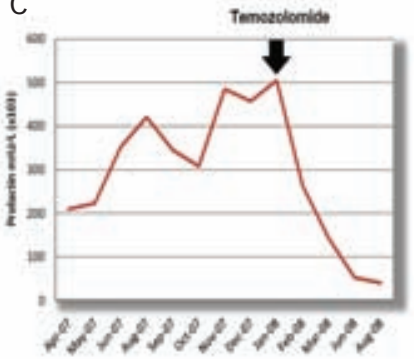

D
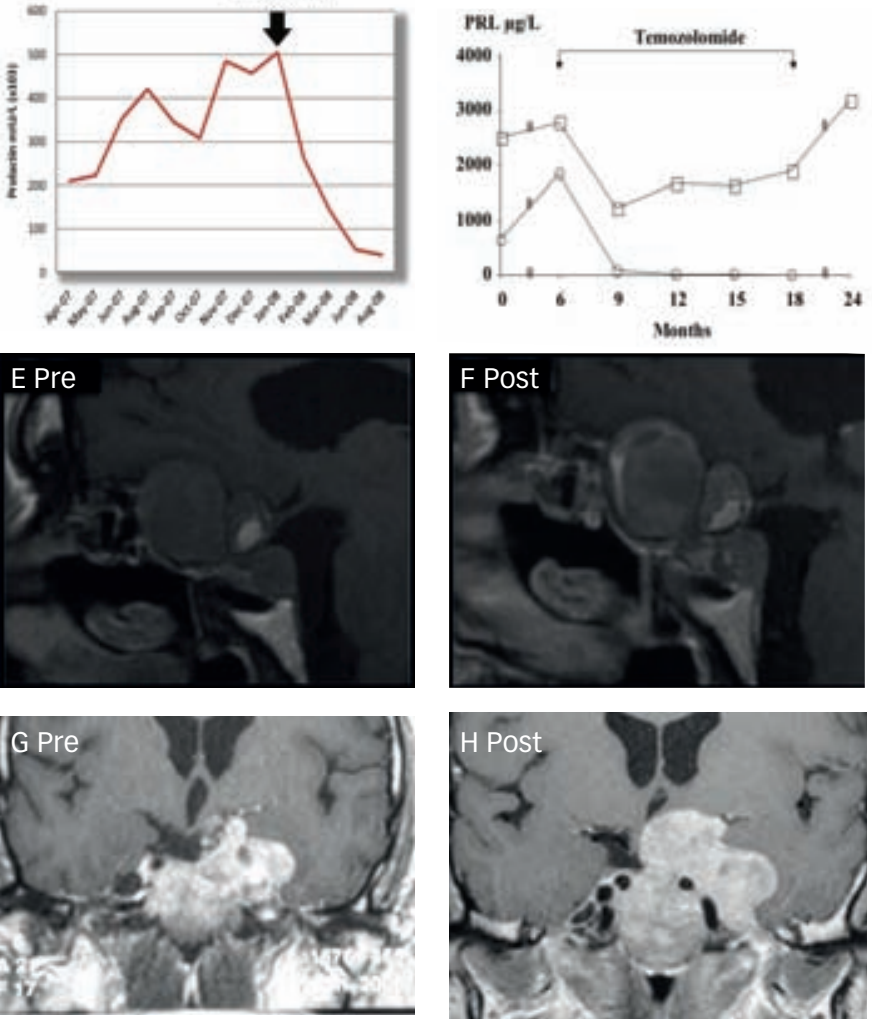

(a) to (c) show a case example of an excellent and sustained response: 42-year-old male with malignant prolactinoma. ${ }^{51}$ (a) and (b) shows radiological images prior to and after 15 cycles of temozolomide. (c) shows a decline in prolactin levels after temozolomide therapy. (d) to (f) show a case example of a stable response: 62-year-old male with aggressive prolactinoma, ${ }^{33}(d)$ shows a square line graph demonstrating initial hormonal response with subsequent plateau as compared with another case illustrating complete hormonal response. (e) and (f) show radiological images prior to and after 12 cycles of temozolomide. $(g)$ and (h) show a case example of progressive disease: 41 year old male with silent adrenocorticotrophic hormone-secreting tumor, ${ }^{; 1}$ radiological images prior to and after eight months of temozolomide therapy.

which temozolomide has been used. More recent case series, each including 6-8 cases regardless of outcome, have contributed to correcting the initial positive publication bias. ${ }^{31-33}$ The clinical spectrum of response to temozolomide is now recognized; whilst the majority demonstrate an excellent and sustained response, stable or transient responses and occasional cases with clear progressive disease are also seen. Figure 2 illustrates this clinical spectrum utilizing cases drawn from the literature.

\section{Patient Characteristics}

Table 1 summarizes the clinical and pathologic characteristics of the published case cohort. Temozolomide has been most commonly used
Table 1: Published Cases - Clinical and Pathologic Characteristics

\begin{tabular}{|c|c|c|c|c|c|c|c|c|c|c|}
\hline $\begin{array}{l}\text { Tumor } \\
\text { Subtype }\end{array}$ & \multicolumn{2}{|c|}{ PRL } & \multicolumn{2}{|c|}{ ACTH } & \multicolumn{2}{|l|}{$\mathrm{NF}$} & \multicolumn{2}{|l|}{$\mathrm{GH}$} & \multicolumn{2}{|c|}{ TOTAL } \\
\hline Total & 19 & & 19 & & 10 & & 2 & & 50 & \\
\hline Carcinoma/ & $\mathrm{Ca}$ & $\mathrm{Ad}$ & $\mathrm{Ca}$ & $\mathrm{Ad}$ & $\mathrm{Ca}$ & $\mathrm{Ad}$ & $\mathrm{Ca}$ & Ad & $\mathrm{Ca}$ & $\mathrm{Ad}$ \\
\hline Adenoma & 8 & 11 & 10 & 9 & 3 & 7 & 0 & 2 & 21 & 29 \\
\hline $\begin{array}{l}\text { Ki67* } \\
\text { (average) }\end{array}$ & \multicolumn{2}{|c|}{$\begin{array}{l}0-60 \% \\
(14.3 \%)\end{array}$} & \multicolumn{2}{|c|}{$\begin{array}{l}0.5-31 \% \\
(10.7 \%)\end{array}$} & \multicolumn{2}{|c|}{$\begin{array}{l}1-20 \% \\
(7.6 \%)\end{array}$} & \multicolumn{2}{|l|}{$4 \%$} & \multicolumn{2}{|c|}{$\begin{array}{l}0-60 \% \\
(11.2 \%)\end{array}$} \\
\hline $\begin{array}{l}\text { Number of } \\
\text { surgeries }\end{array}$ & \multicolumn{2}{|c|}{$1-6(2.7)$} & \multicolumn{2}{|c|}{$1-5(2.6)^{\#}$} & \multicolumn{2}{|c|}{$0-6(2.4)^{\#}$} & \multicolumn{2}{|c|}{$2-6(4)$} & \multicolumn{2}{|c|}{$0-6(2.7)$} \\
\hline
\end{tabular}

(average)

ACTH = adrenocorticotrophic hormone-secreting; $\mathrm{GH}$ = growth hormone-secreting: $N F=$ non-functioning; $P R L$ = prolactin-secreting. *Ki67 reported for: 14 PRL, 14 ACTH, $8 \mathrm{NF}$ and $1 \mathrm{GH}$ tumors. "Not reported in one case.

for prolactin (PRL)-secreting (19 cases) and ACTH-secreting (19 cases) carcinomas and aggressive adenomas, reflecting the predominance of these tumor types amongst cases of aggressive pituitary tumors. There are 10 non-functioning (NF) pituitary tumors in the cohort and only two growth hormone $(\mathrm{GH})$-secreting tumors. Male patients with aggressive pituitary tumors form the majority of reported cases (29/42). The average patient age is 46.4 years, range $13-72$. Whilst the range of measured Ki67 indices varies widely between cases, the aggressive nature of the tumors comprising this cohort is reflected in the high average Ki67 of $11.2 \%$. In the vast majority of cases, treatment with temozolomide is used as salvage therapy. Patients have typically undergone multimodal therapy, often including several surgeries and one or more courses of radiotherapy over several years. Tumors also display resistance to medical therapy with dopamine agonists and somatostatin analogs, although these agents are often continued whilst the patient is receiving temozolomide.

Perhaps reflecting the mounting international experience and encouraging evidence of efficacy is the recent report by Whitelaw et al. of use of temozolomide in a non-salvage setting. They successfully used temozolomide as a means of deferring radiotherapy in a pediatric patient and facilitated subsequent radiotherapy in another patient with chiasmal compression by effecting tumor shrinkage. ${ }^{34}$

\section{Temozolomide Treatment Regimes}

Patients are typically chemotherapy-naïve when temozolomide treatment is commenced. On the whole, temozolomide has been given as monotherapy. Significant side effects were encountered when temozolomide was commenced in combination with interferon or thalidomide, ${ }^{25,32}$ however, in another two cases it was well tolerated when used with capecitabine or pasireotide. ${ }^{35,36}$ In two cases demonstrating progressive disease whilst on temozolomide therapy alone, alternative chemotherapeutic agents have been added with no additional benefit; carmustine in one case and carboplatin in another case. ${ }^{31}$ The standard temozolomide-dosing regime of $150-200 \mathrm{mg} / \mathrm{m}^{2}$ /day for five days over 28 days is most commonly used. In the case series reported by Bush et al., a dose-dense regime was employed $\left(75 \mathrm{mg} / \mathrm{m}^{2} /\right.$ day for 21 days with seven days off). The most variable aspect in the temozolomide treatment regime between cases is therapy duration. More commonly, patients receive a prescribed course of 6-12 months of temozolomide. Early progressive disease has necessitated cessation as early as two or 
Figure 3: Temozolomide Response by Pituitary Tumor Subtype

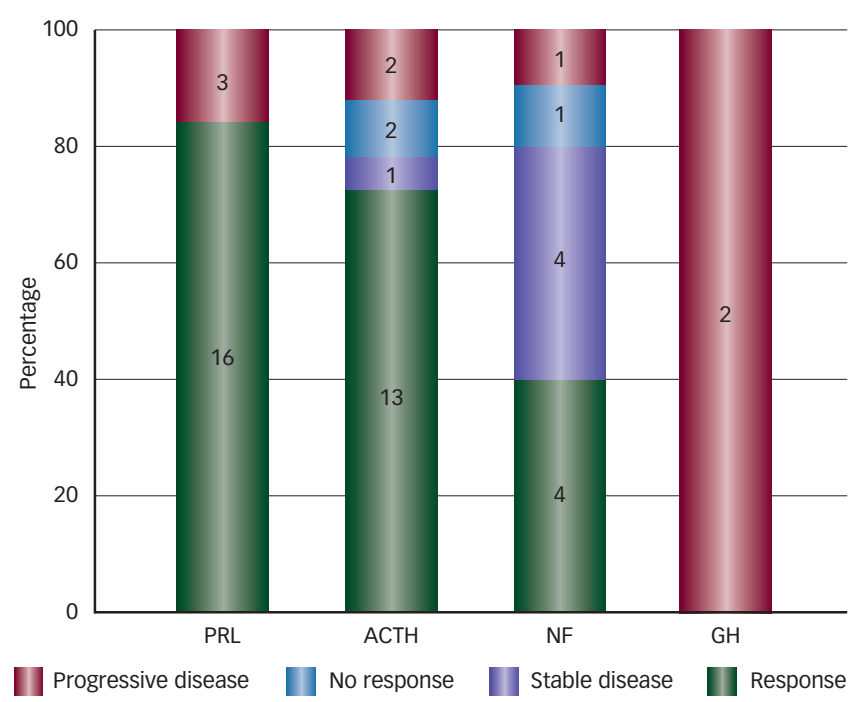

$A C T H=$ adrenocorticotrophic hormone-secreting tumor; $P R L=$ prolactin-secreting tumor; $\mathrm{GH}=$ growth hormone-secreting tumor; NF = non-functioning tumor.

three months, whilst other cases demonstrating good response continue on temozolomide beyond 24 months and in one patient five years. ${ }^{27,31,33,37}$

\section{Adverse Effects}

Temozolomide-related adverse effects have been reported in 22 of the 50 cases. Nausea, fatigue, headaches and thrombocytopenia are most frequently described and typically mild. However, fatigue contributed to a decision to cease treatment in three cases. Dose reduction or extension in dosing interval has permitted continuation of temozolomide in patients developing mild myelosuppression. Severe adverse events necessitating temozolomide cessation have occurred in two cases: in one patient agranulocytosis and the other sensorineural hearing loss.

\section{Response to Temozolomide}

Overall, 33 of 49 patients (67 \%) have demonstrated a response to temozolomide therapy. Both adenomas and carcinomas may show response. Figure 3 illustrates temozolomide response by pituitary tumor subtype. PRL and ACTH tumors had a higher response rate (84 and $72 \%$, respectively) than NF tumors (40\%), although a significant proportion of NF tumors demonstrated stable disease (40\%). Invariably a clinical response predicted a subsequent radiological tumoral response, and in functioning tumors, a hormonal response as well. In just one case, a hormonal response was not accompanied by any clinical improvement or tumor shrinkage. ${ }^{33}$ When it occurs, response is typically seen within the first three months, leading some to propose a three-month treatment trial. ${ }^{31}$ However, maximal response is often only seen after 10-12 cycles of treatment. ${ }^{30,33}$ In addition, stable disease may be a valuable outcome; there is a low rate (16\%) of reported tumor progression during temozolomide treatment.

The application of traditional RECIST (Response Evaluation Criteria In Solid Tumors) radiological criteria to report degree of tumor shrinkage (partial response $>30 \%$ decrease in tumor diameter; complete response disappearance of tumor) may significantly underestimate the degree of tumor shrinkage observed in many responders. Most pituitary tumors
Figure 4: Temozolomide Response and MGMT Expression by Immunohistochemistry

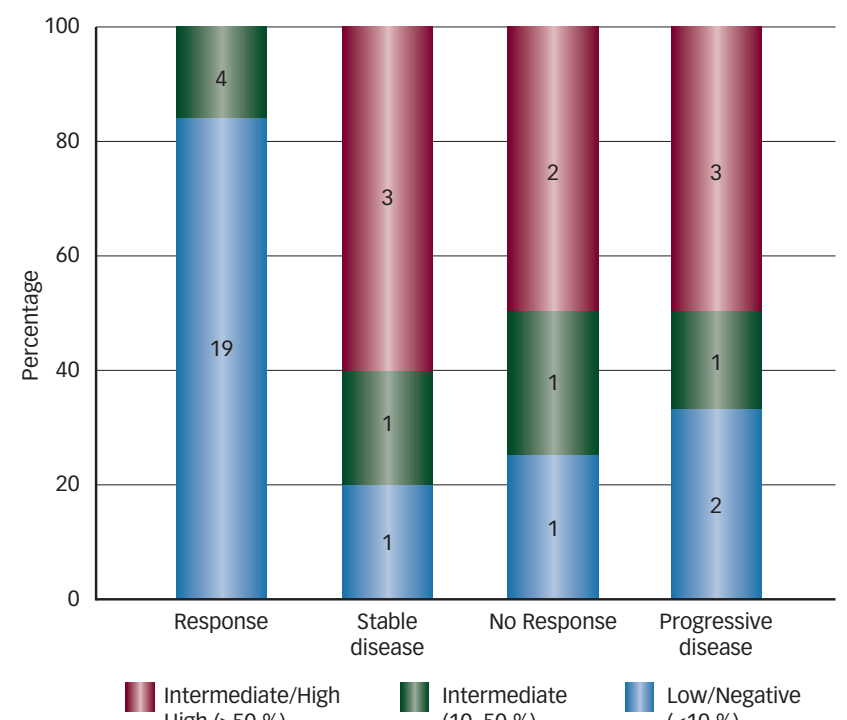

MGMT = 06-methylguanine-DNA methyltransferase

that respond to temozolomide show a $>50 \%$ tumor shrinkage, in some cases $>80 \%$, and in three cases complete response was seen in metastatic lesions. ${ }^{30-33,35,37}$ Similarly, hormonal responses can be remarkable, often occurring earlier and to a greater degree than radiological responses, and with more than half of cases demonstrating biochemical normalization. Hormonal response may translate into significant clinical benefits most obviously for ACTH or GH tumors.

Another notable feature seen in a number of pituitary tumors is a prolonged and sustained response following temozolomide cessation, with no tumor regrowth for as long as three years. ${ }^{24,30,33}$ Morphologic change in the tumor following response has been described in one case, with evidence of increased differentiation, may contribute to this phenomenon. ${ }^{38}$ However, eventual tumor regrowth is increasingly described. ${ }^{33,39,40}$ Unfortunately, a second course of temozolomide in the setting of tumor regrowth has to date not been effective ${ }^{40}$ (and personal communication with William Drake and Richard Ross). It is possible that a subset of tumor cells originally resistant to temozolomide explain eventual tumor regrowth. Alternatively, the development of additional acquired genetic alterations in the tumor may drive temozolomide resistance. Murakami et al. recently eloquently described transformation of an atypical prolactinoma into a pituitary carcinoma in association with acquired temozolomide resistance and the loss of MutS Homolog 6 (MSH6) (a MMR protein).

In patients with non-response or tumor progression, various alternative treatments have been tried. Trials of alternative chemotherapeutic agents have been largely unsuccessful. ${ }^{33,35,40}$ Bevacizumab (vascular endothelial growth factor [VEGF] inhibitor) has been used successfully in one case, ${ }^{41}$ whilst pasireotide has also been trialed. . $^{33}$

\section{MGMT Status and Response to Temozolomide Therapy in Pituitary Tumors}

MGMT is an evolutionarily conserved gene, responsible for repairing 06MeG cytotoxic adducts that may arise as a result of endogenous DNA 
Figure 5: MGMT Expression by Immunohistochemistry and Temozolomide Response

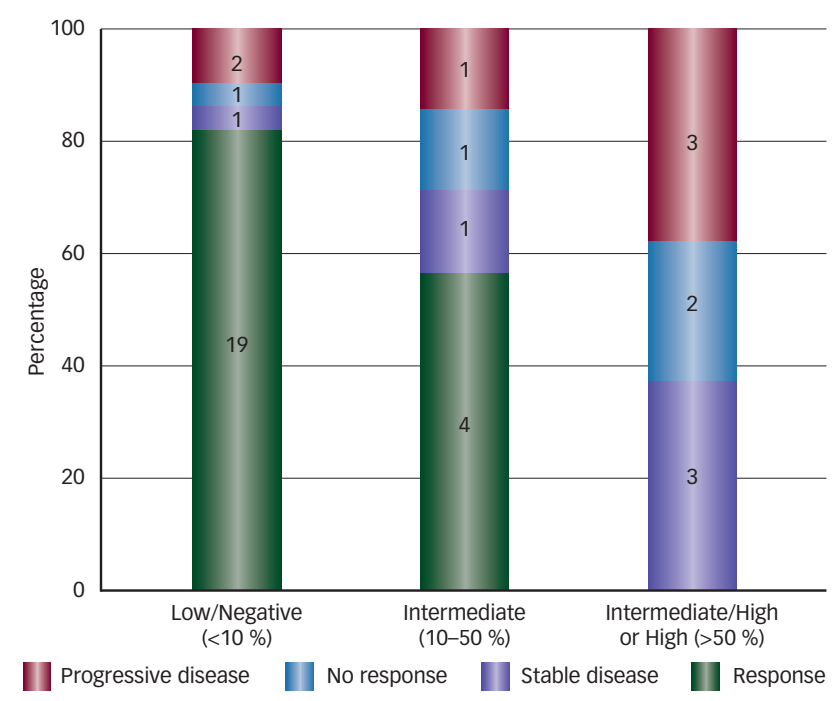

MGMT = 06-methylguanine-DNA methyltransferase

damage, or exogenously via ionizing radiation or chemotherapeutic agents such as temozolomide. 11142 Low MGMT expression and temozolomide efficacy has been repeatedly demonstrated in brain tumors, but also seen in melanoma and neuroendocrine tumors. ${ }^{43-47}$ MGMT expression as assessed by immunohistochemistry has been studied in 38 of the published pituitary cases. Response to temozolomide has only been described in association with low or negative MGMT expression (83\%) or intermediate expression (17\%) (see Figure 4). However, stable or progressive disease occurred in association with a wide range of MGMT expression (see Figure 4). As illustrated in Figure 5, low or negative MGMT expression predicts a high chance of response (83\%), although there were two cases with low expression demonstrating clearly progressive disease. In addition, response to temozolomide has not been described in association with $>50 \%$ MGMT expression. These results strongly suggest MGMT expression is the primary determinant of response, although in some instances other resistance mechanisms clearly operate. In their recent review, Whitelaw et al., found that pituitary tumors with low or negative MGMT expression were 9.35 times more likely to respond to temozolomide than tumors with intermediate or high MGMT expression. ${ }^{34}$

The presence of MGMT promoter methylation has been linked with response to temozolomide in glioblastomas.44,45,48 In fact, MGMT immunohistochemistry may be unreliable in glioblastomas because of difficulty in distinguishing MGMT expression in neoplastic from non-neoplastic inflammatory cells, the latter extensively found in glioma specimens. ${ }^{49}$ However, MGMT methylation status does not correlate with temozolomide response in pituitary tumors. MGMT methylation analysis has been reported in 20 cases: three of five methylated tumors and nine of 15 unmethylated tumors were associated with treatment response..$^{50}$ Progressive disease has also been seen in cases demonstrating promoter methylation. Furthermore, MGMT promoter methylation is not the predominant mechanism causing loss of MGMT expression in pituitary tumors: of 10 cases with low MGMT expression by immunohistochemistry only four were found to be methylated..$^{50}$ The mechanism responsible for loss of MGMT expression in pituitary tumors remains unknown. MGMT gene mutations have not been detected in pituitary tumors. ${ }^{51}$ Loss of heterozygosity at $10 \mathrm{q} 26$, the region containing the MGMT gene, has also not been identified in a small number of Iow MGMT-expressing pituitary tumors. ${ }^{51}$

\section{MGMT and Pituitary Tumor Biology}

The observation of a pituitary tumor subtype difference in response to temozolomide, as well as differential expression of MGMT across pituitary tumors, have led to examination of MGMT expression in larger archived cohorts of unselected pituitary tumors. Overall in one such cohort, $13 \%$ of pituitary tumors were found to have low MGMT expression (defined as $<10 \%$ ), and $28 \%$ and $59 \%$ with intermediate (10-90\%) and high (>90\%) MGMT expression, respectively. ${ }^{51}$ There is a clear greater propensity for prolactin-producing tumors towards low MGMT expression, which is likely to account for the higher response rate of prolactinomas to temozolomide therapy. ${ }^{34,51,52}$ Some studies have not found significantly increased differences in MGMT expression between invasive and non-invasive pituitary tumors. ${ }^{51,52}$ However, other studies have reported a higher incidence of low MGMT expression amongst more aggressive pituitary tumor subtypes, including invasive Crooke's cell adenoma and silent subtype 3 pituitary adenomas. ${ }^{37,53}$ A higher occurrence of low MGMT expression has been reported amongst primary surgical specimens of NF pituitary adenomas showing subsequent tumor regrowth. Furthermore, those primary NF pituitary adenomas with Iow MGMT expression had a shorter interval until re-operation. ${ }^{54}$ These limited studies do suggest a role for MGMT in pituitary tumor biology.

\section{Future Directions}

The identification of temozolomide as the first chemotherapeutic agent with significant efficacy in aggressive pituitary tumors represents a major advance in management. It is hoped the next few years will see further clarity develop in delineating the role of temozolomide in the treatment of pituitary tumors. There is excitement about the potential for its use earlier in the treatment algorithm, but also justifiable concern about the lack of long-term safety data in particular with regard to secondary malignancies.

One of the more pressing questions relates to the optimal length of temozolomide therapy. Response is invariably seen within three months, and this appears a good time frame for a therapeutic trial. In responding tumors, maximal benefit is most commonly seen within the first 12 months, but whether longer treatment would affect more prolonged responses is unknown. Various combination therapies may offer the possibility of enhanced clinical benefit, particularly in non-responding cases or tumors with high MGMT expression. MGMT inhibitors, such as 06-benzylguanine, in combination with temozolomide, have had limited success in resistant gliomas. ${ }^{55}$ Synergistic chemotherapeutic effect has been described for temozolomide in combination with bevacizumab, and there is interest in inhibition of poly (ADP-ribose) polymerase (PARP) (another DNA repair enzyme) and potentiation of temozolomide efficacy. ${ }^{56,57}$

The use of temozolomide as salvage therapy for aggressive pituitary tumors is generally well accepted, including recommendation in recent Endocrine Society Clinical Guidelines for consideration of its use for malignant prolactinomas. ${ }^{58}$ The possibility of utilizing temozolomide earlier in the treatment paradigm has recently been raised. Whitelaw 
et al. have successfully used temozolomide in order to achieve tumor shrinkage in a tumor compressing the optic chiasm prior to administering radiotherapy. They also described temozolomide treatment in a pediatric patient as a means of deferring radiotherapy. ${ }^{34}$ Temozolomide has demonstrated radiosensitizing properties, and potential concurrent administration of temozolomide and radiotherapy may provide increased benefit. ${ }^{11}$

Whilst MGMT appears to play a primary role in determining response to temozolomide in pituitary tumors, a better understanding of additional resistance mechanisms is needed. Reports in glioblastoma have implicated high epidermal growth factor receptor expression and upregulation of stem cell gene pathways in MGMT-independent temozolomide resistance. ${ }^{59,60}$ In the author's opinion, MGMT expression as determined by immunohistochemistry does provide guidance for more effective use of temozolomide in pituitary tumors. However, the wider application of MGMT immunohistochemistry into clinical practice requires the development of standardised scoring methods. Finally, the role of MGMT in pituitary tumorigenesis and the mechanism(s) responsible for loss of MGMT expression in pituitary tumors remain to be elucidated.

Prospective clinical trials would provide the ideal platforms to properly address many of the uncertainties surrounding the use of temozolomide in aggressive pituitary tumors. International collaborations will become immensely important in driving forward advancements in this challenging area of clinical practice.
1. Ezzat S, Asa SL, Couldwell WT, et al., The prevalence of pituitary adenomas: a systematic review, Cancer, 2004:101:613-9.

2. Daly AF, Rixhon M, Adam C, et al., High prevalence of pituitary adenomas: a cross-sectional study in the province of Liege, Belgium, I Clin Endocrinol Metab, 2006;91:4769-75.

3. Fernandez A, Karavitaki N, Wass JA, Prevalence of pituitary adenomas: a community-based, cross-sectional study in Banbury (Oxfordshire, UK), Clin Endocrinol (Oxf). 2010;72:377-82.

4. Kaltsas GA, Nomikos P, Kontogeorgos G, et al., Clinical review: Diagnosis and management of pituitary carcinomas, I Clin Endocrinol Metab, 2005;90:3089-99.

5. Sautner D, Saeger W, Invasiveness of pituitary adenomas, Pathol Res Pract, 1991;187:632-6.

6. Scheithauer BW, Kovacs KT, Laws ER Jr, Randall RV, Pathology of invasive pituitary tumors with special reference to functiona classification, J Neurosurg, 1986;65:733-44.

7. Lloyd RV, Kovacs K, Young WF, Pituitary Tumors, in: DeLellis RA, Lloyd RV, Heitz PU, Eng C (eds), World Health Organization Classification of Tumours, Pathology and Genetics of Tumours of Endocrine Organs, Lyon: IARC Press: 2004;10-47.

8. Zada G, Woodmansee WW, Ramkissoon S, et al., Atypical pituitary adenomas: incidence, clinical characteristics, and implications, I Neurosurg, 2011;114:336-44.

9. Pernicone PJ, Scheithauer BW, Sebo TJ, et al., Pituitary carcinoma: a clinicopathologic study of 15 cases, Cancer 1997:79:804-12.

10. Neyns B, Tosoni A, Hwu WJ, Reardon DA, Dose-dense temozolomide regimens: antitumor activity, toxicity, and immunomodulatory effects, Cancer, 2010;116:2868-77.

11. Marchesi F, Turriziani M, Tortorelli G, et al., Triazene compounds: mechanism of action and related DNA repair systems, Pharmacol Res, 2007;56:275-87.

12. Kaina B, Ziouta A, Ochs K, Coquerelle T, Chromosomal instability, reproductive cell death and apoptosis induced by O6-methylguanine in Mex-, Mex+ and methylation-tolerant mismatch repair compromised cells: facts and models, Mutat Res, 1997;381:227-41.

13. Newlands ES, Blackledge GR, Slack JA, et al., Phase I trial of temozolomide (CCRG 81045: M\&B 39831: NSC 362856), Br J Cancer, 1992;65:287-91.

14. Lashkari HP, Saso S, Moreno L, et al., Using different schedules of Temozolomide to treat low grade gliomas: systematic review of their efficacy and toxicity, J Neurooncol, 2011;105:135-47.

15. Stupp R, Mason WP, van den Bent MJ, et al. Radiotherapy plus concomitant and adjuvant temozolomide for glioblastoma, N Eng/ I Med, 2005;352:987-96

16. Middleton MR, Grob JJ, Aaronson N, et al., Randomized phase III study of temozolomide versus dacarbazine in the treatmen of patients with advanced metastatic malignant melanoma J Clin Oncol, 2000:18:158-66.

17. Hau P, Koch D, Hundsberger $T$, et al., Safety and feasibility of long-term temozolomide treatment in patients with high-grade glioma, Neurology, 2007;68:688-90.

18. Khasraw $M$, Bell $D$, Wheeler $H$, Long-term use of temozolomide could you use temozolomide safely for life in gliomas? J Clin Neurosci, 2009;16:854-5.

19. Kim SJ, Park TS, Lee ST, et al., Therapy-related myelodysplastic syndrome/acute myeloid leukemia after treatment with temozolomide in a patient with glioblastoma multiforme Ann Clin Lab Sci, 2009:39:392-8.

20. George BJ, Eichinger JB, Richard TJ, A rare case of aplastic anemia caused by temozolomide, South Med J, 2009;102:974-6

21. Neyns B, Cordera S, Joosens E, Pouratian N, Non-Hodgkin's lymphoma in patients with glioma treated with temozolomide, J Clin Oncol, 2008;26:4518-9.

22. Ekeblad S, Sundin A, Janson ET, et al., Temozolomide as monotherapy is effective in treatment of advanced malignant neuroendocrine tumors, Clin Cancer Res, 2007;13:2986-91.
23. Guilleminault $L$, Carré $P$, Diot $P$, Hypersensitivity pneumonitis associated with temozolomide, Eur Respir J, 2009:34:526.

24. Lim S, Shahinian H, Maya MM, et al., Temozolomide: a novel treatment for pituitary carcinoma, Lancet Oncol, 2006:7:518-20.

25. Fadul $C E$, Kominsky AL, Meyer LP, et al., Long-term response of pituitary carcinoma to temozolomide. Report of two cases, I Neurosurg, 2006;105:621-6.

26. Syro LV, Uribe $H$, Penagos LC, et al., Antitumour effects of temozolomide in a man with a large, invasive prolactin-producing pituitary neoplasm, Clin Endocrinol (Oxf), 2006;65:552-3.

27. Neff $L M$, Weil $M$, Cole $A$, et al., Temozolomide in the treatment of an invasive prolactinoma resistant to dopamine agonists, Pituitary, 2007;10:81-6.

28. Syro LV, Scheithauer BW, Ortiz LD, et al., Effect of temozolomide in a patient with recurring oncocytic gonadotrophic pituitary adenoma, Hormones (Athens), 2009;8:303-6.

29. Moyes VJ, Alusi G, Sabin HI, et al., Treatment of Nelson's syndrome with temozolomide, Eur J Endocrinol, 2009:160:115-9.

30. Hagen C, Schroeder HD, Hansen S, et al., Temozolomide treatment of a pituitary carcinoma and two pituitary macroadenomas resistant to conventional therapy, Eur J Endocrinol, 2009;161:631-7.

31. Raverot G, Sturm N, de Fraipont F, et al., Temozolomide treatment in aggressive pituitary tumors and pituitary carcinomas: a French multicenter experience, $J$ Clin Endocrinol Metab, 2010:95(10):4592-9.

32. Bush ZM, Longtine JA, Cunningham T, et al., Temozolomide treatment for aggressive pituitary tumors: correlation of clinica outcome with 06-methylguanine methyltransferase (MGMT) promoter methylation and expression, J Clin Endocrinol Metab, 2010;95(11):E280-90.

33. Losa M, Mazza E, Terreni MR, et al., Salvage therapy with Temozolomide in patients with aggressive or metastatic pituitary adenomas: experience in six cases, Eur J Endocrinol, 2010;163(6):843-51.

34. Whitelaw B, Dworakowska D, Thomas NW, et al. Temozolomide in the management of dopamine agonistresistant prolactinomas, Clin Endocrinol (0xf), 2012;76(6):877-86.

35. Thearle MS, Freda PU, Bruce JN, et al., Temozolomide (Temodar ${ }^{\circledR}$ ) and capecitabine (Xeloda ${ }^{\circledR}$ ) treatment of an aggressive corticotroph pituitary tumor, Pituitary, 2011:14(4):418-24.

36. Bode $\mathrm{H}$, Seiz M, Lammert $\mathrm{A}$, et al., SOM230 (Pasireotide) and Temozolomide achieve sustained control of tumour progression and ACTH secretion in pituitary carcinoma with widespread metastases, Exp Clin Endocrinol Diabetes, 2010;118:760-3.

37. Takeshita A, Inoshita N, Taguchi M, et al., High incidence of low 06-methylguanine DNA methyltransferase (MGMT) expression in invasive macroadenomas of Cushing's disease, Eur J Endocrinol 2009;161(4):553-9.

38. Kovacs K, Horvath E, Syro LV, et al., Temozolomide therapy in a man with an aggressive prolactin-secreting pituitary neoplasm: Morphological findings, Hum Pathol, 2007;38:185-9.

39. Mohammed S, Kovacs K, Mason W, et al., Use of temozolomide in aggressive pituitary tumors: case report, Neurosurgery, 2009;64:E773-4; discussion E4.

40. Murakami M, Mizutani A, Asano S, et al., A mechanism of acquiring temozolomide resistance during transformation of atypical prolactinoma into prolactin-producing pituitary carcinoma: case report, Neurosurgery, 2011;68:E1761-7: discussion E7.

41. Moshkin O, Syro LV, Scheithauer BW, et al., Aggressive silent corticotroph adenoma progressing to pituitary carcinoma: the role of temozolomide therapy, Hormones (Athens), 2011;10:162-7.

42. Hoeijmakers JH, DNA damage, aging, and cancer, N Eng/ J Med, 2009;361:1475-85.

43. Friedman HS, McLendon RE, Kerby $T$, et al., DNA mismatch repair and 06-alkylguanine-DNA alkyltransferase analysis and response to Temodal in newly diagnosed malignant glioma, J Clin Oncol, 1998:16:3851-7.

44. Esteller M, Garcia-Foncillas I, Andion E, et al., Inactivation of the DNA-repair gene MGMT and the clinical response of gliomas to alkylating agents, N Engl J Med, 2000;343:1350-4.

45. Hegi ME, Diserens AC, Gorlia T, et al., MGMT gene silencing and benefit from temozolomide in glioblastoma, N Engl J Med, 2005;352:997-1003.

46. Augustine CK, Yoo JS, Potti A, et al., Genomic and molecular profiling predicts response to temozolomide in melanoma, Clin Cancer Res, 2009:15:502-10.

47. Kulke $M H$, Hornick JL, Frauenhoffer $\mathrm{C}$, et al., O6-methylguanine DNA methyltransferase deficiency and response to temozolomide-based therapy in patients with neuroendocrine tumors, Clin Cancer Res, 2009;15:338-45.

48. Cao VT, Jung TY, Jung $\mathrm{S}$, et al., The correlation and prognostic significance of MGMT promoter methylation and MGMT protein in glioblastomas, Neurosurgery, 2009;65:866-75; discussion 75.

49. Rodriguez FJ, Thibodeau SN, Jenkins RB, et al., MGMT immunohistochemical expression and promoter methylation in human glioblastoma, Appl Immunohistochem Mol Morphol, 2008;16:59-65

50. McCormack Al, Wass JA, Grossman AB, Aggressive pituitary tumours: the role of temozolomide and the assessment of MGMT status, Eur I Clin Invest, 2011;41:1133-48.

51. McCormack Al, MCDonald KL, Gill AJ, et al., Low O6methylguanine-DNA methyltransferase (MGMT) expression and response to temozolomide in aggressive pituitary tumours, Clin Endocrinol (Oxf), 2009;71:226-33.

52. Lau Q, Scheithauer B, Kovacs K, et al., MGMT immunoexpression in aggressive pituitary adenoma and carcinoma, Pituitary, 2010;13(4):367-79.

53. Fealey ME, Scheithauer BW, Horvath E, et al., MGMT immunoexpression in silent subtype 3 pituitary adenomas: possible therapeutic implications, Endocr Pathol, 2010;21(3):161-5.

54. Widhalm G, Wolfsberger S, Preusser M, et al., O(6)methylguanine DNA methyltransferase immunoexpression in nonfunctioning pituitary adenomas: are progressive tumors potential candidates for temozolomide treatment?, Cancer, 2009;115:1070-80.

55. Quinn JA, Jiang SX, Reardon DA, et al., Phase II trial of temozolomide plus 06-benzylguanine in adults with recurrent, temozolomide-resistant malignant glioma, Clin Oncol, 2009;27:1262-7.

56. Kulke MH, Earle CC, A phase II study of temozolomide and bevacizumab in patients with advanced neuroendocrine tumors, Journal of Clinical Oncology, 2006 ASCO Annual Meeting Proceedings Part I. Vol 24, No. 18S (June 20 Supplement), 2006:4044.

57. Palma JP, Rodriguez LE, Bontcheva-Diaz VD, et al., The PARP inhibitor, ABT-888 potentiates temozolomide: correlation with drug levels and reduction in PARP activity in vivo, Anticancer Res, 2008:28:2625-35.

58. Melmed S, Casanueva FF, Hoffman AR, et al., Diagnosis and treatment of hyperprolactinemia: an Endocrine Society clinica practice guideline, J Clin Endocrinol Metab, 2011;96:273-88.

59. Murat A, Migliavacca E, Gorlia T, et al., Stem cell-related "self-renewal" signature and high epidermal growth factor receptor expression associated with resistance to concomitant chemoradiotherapy in glioblastoma, j Clin Oncol, 2008:26:3015-24.

60. Gaspar N, Marshall L, Perryman L, et al., MGMT-independent temozolomide resistance in pediatric glioblastoma cells associated with a PI3-kinase-mediated HOX/stem cell gene signature, Cancer Res, 2010;70:9243-52.

61. Kovacs K, Scheithauer BW, Lombardero M, et al, MGMT immunoexpression predicts responsiveness of pituitary tumors to temozolomide therapy, Acta Neuropathol, 2008;115:261-2. 